\title{
ORT.03 - Comparison between different automated methodologies for the identification of gram-positive rods isolated from clean rooms
}

\author{
Luciana Veloso da Costa ${ }^{1 *}$; Erica Louro da Fonseca ${ }^{1}$; Natália Pedra Gonçalves ${ }^{1}$; Cristhiane Moura \\ Falavina $^{1}$; Adriana Marques Frazão ${ }^{1}$; Joyce Modesto de Andrade ${ }^{1}$; Verônica Viana Vieira ${ }^{2}$. \\ 1Fiocruz/Bio-Manguinhos; \\ 2Fiocruz/IOC.
}

Introduction: One of the major risks associated with drugs and immunobiologicals production is microbial contamination. Therefore, an environmental monitoring program is essential to ensure that aseptic production areas are maintained under appropriate control levels. To be efficient, it must contain an adequate identification level of isolated microorganisms, because the information provided may be extremely relevant in investigating sources of contamination of products or processes. Sporulated Gram-positive rods (SGPR) are among the main contaminant groups of productive clean areas from Bio-Manguinhos in 2016 and 2017. Due to spore resistance, SGPR's are not easily eliminated. SGPR identification by classical methods is particularly difficult because of similarities between closely related species that share the same morphological and biochemical patterns. The pharmaceutical industry has adopted automated identification systems, due to greater rigidity in regulatory requirements, regarding microbial identification. However, many identification systems have databases directed to medical microorganisms, being more limited considering pharmaceutical and industrial environmental microorganisms, which makes it difficult to obtain results at species level.

Objective: The aim of this study was to compare three automated methodologies: VITEK ${ }^{\circledast} 2$ Compact (bioMérieux), VITEK ${ }^{\circledast}$ MS RUO (bioMérieux) and MicroSEQ ${ }^{\circledR}$ Full Gene $16 \mathrm{~S}$ rDNA (Thermo Fisher Scientific) in SGPR isolated from Bio-Manguinhos productive áreas identification.

Methodology: 98 strains of SGPR were isolated from Bio-Manguinhos clean rooms from 2016 to 2017 . These strains were analyzed by three different methodologies: biochemical identification by VITEK ${ }^{\circledast} 2$ Compact; molecular identification through proteome profile analysis generated by VITEK ${ }^{\circledast}$ MS RUO; and molecular identification by sequencing the $16 \mathrm{~S}$ rDNA gene, through MicroSEQ ${ }^{\circledR}$ Full Gene $16 \mathrm{~S}$ rDNA kit.

Results: For 98 isolates, $45.9 \%$ were identified at the species level; $19.4 \%$ at the genus level; $21.4 \%$ of the results were inconclusive and $13.3 \%$ were not identified by VITEK 2. Identification by VITEK $^{\circledast}$ MS RUO showed $26.5 \%$ of isolates identified at the species level; $38.8 \%$ at the genus level and $34.7 \%$ were not identified. Identification by sequencing of the $16 \mathrm{~S}$ rDNA gene provided $44.6 \%$ of the results at the species level; $54.1 \%$ at the genus level and $1.3 \%$ of the results were inconclusive. Identification by sequencing has not yet been completed.

Conclusion: The highest percentage of identified microorganisms was obtained by sequencing the $16 \mathrm{~S}$ rDNA gene. However, other housekeeping genes should be tested to enhance methodological performance for species-level identification. On the other hand, VITEK MS provides lower time and cost results as long as a robust database based on proteomic spectra is built. By combining the two methodologies, housekeeping genes sequencing enables the identification of SGPR species, whose spectra can be inserted in the database, configuring an effective identification tool in a reduced time and cost.

Keywords: VITEK MS; 16S rDNA; clean rooms 